\title{
CATÁLOGO Y ATLAS DE LAS ARAÑAS DE LA FAMILIA ANYPHAENIDAE EN LA PENÍNSULA IBÉRICA E ISLAS BALEARES
}

\author{
C. Urones 1
}

\begin{abstract}
RESUMEN
El catálogo incluye las cuatro especies de Anyphaenidae (Araneae) conocidas en la Península Ibérica y Baleares. Para cada especie se recogen, ordenadas por provincias, todas las citas bibliográficas y se incluyen nuevas localidades correspondientes al material de la colección del Museo Nacional de Ciencias Naturales de Madrid, así como ejemplares procedentes de la colección de la autora, que amplían considerablemente el área de distribución conocida para cada una de ellas. Se da el atlas provisional de distribución de las especies de Anyphaenidae para el territorio de estudio sobre mapas de cuadrícula U.T.M. de $50 \mathrm{~km}$ de lado. Y se aportan, además, datos sobre su hábitat y ciclos de vida.
\end{abstract}

Palabras clave: Arañas, Araneae, Anyphaenidae, Distribución Geográfica, España, Portugal.

\section{ABSTRACT \\ Catalogue and atlas of the Spider Family Anyphaenidae of Iberian Peninsule and Balearic Islands}

A catalogue including the four know especies of Anyphaenidae from the Iberian Peninsula and Balearic Islands is provided. For each species, a list of all the localities, arranged according to provinces, obtained from literature references is presented. New localities are also given, both coming from "the Museo Nacional de Ciencias Naturales in Madrid" and the authoress collections. A provisional distribution atlas of Anyphaenidae species for the studied area is also provided, together with distribution maps for each species. Some data concerning habitat and life cycle are given.

Key words: Spiders, Araneae, Anyphaenidae, Geographical Distribution, Spain, Portugal.

\section{Introducción}

Las arañas, debido a su número y a sus hábitos depredadores, constituyen un grupo de gran interés en el equilibrio de los ecosistemas. A pesar de ello pocos investigadores se ocupan de tan interesante grupo y la distribución geográfica de las arañas ibéricas está muy mal conocida.
En este artículo, continuación del dedicado a la familia Philodromidae (Urones, 1995), por lo que sigue su misma estructura, hemos pretendido reunir todas las citas de Anyphaenidae existentes en el ámbito ibero-balear y aportar datos nuevos que amplian mucho el área de distribución conocida para estas especies (Urones et al., 1995). No se menciona Andorra pues no existe ninguna cita de

1 Departamento de Didáctica Matemáticas y Ciencias Experimentales. Facultad de Educación. Universidad de Salamanca. Paseo de Canalejas, 169. 37008 Salmanca. 
arañas de esta familia en ese país. Los ejemplares estudiados proceden de la colección del Museo Nacional de Ciencias Naturales de Madrid $(\mathrm{MNCN})$ y de la colección de la autora, fruto de diversas prospecciones peninsulares. Al reflejar todos estos datos en mapas de distribución se consigue una mejor racionalización de toda la información que se posee hasta la actualidad.

Con la confección de este trabajo pretendemos aportar una herramienta imprescindible para el desarrollo del conocimiento biológico en nuestro país, ya que sin estos trabajos básicos difícilmente se podrán abordar otros aspectos biológicos o corológicos de los que puedan derivarse acciones encaminadas a la protección y conservación de la naturaleza.

Aunque los Anyphaenidae, a lo largo del tiempo, han ocupado diferentes posiciones en las clasificaciones aracnológicas hoy, debido a la posición del estigma traqueal, la composición de los fascículos unguinales de los tarsos y la extensión de su sistema traqueal, nadie duda en concederles el rango de familia independiente. Por el contrario, en cuanto a su origen filogenético hay discrepancias, para Lehtinen (1967) los anifaénidos derivan de los araneomorfos Amaurobiformes, mientras para Coddington \& Levi (1991) basándose en estudios cladísticos los sitúan muy próximos filogenéticamente a los Clubionidae y Salticidae.

La familia Anyphaenidae comprende en la fauna mundial más de 30 géneros y 500 especies (Dondale \& Redner, 1982; Brignoli, 1983; Platnick, 1989, 1993; Brescovit, 1993) presentes fundamentalmente en Sudamérica. De estos géneros sólo hay 1 Holártico-Neotropical: Anyphaena, 3 son Neártico-Neotropical: Aysha Keyserling, 1891, Wulfila O. Pickard-Cambridge, 1895 e Hibana Brescovit, 1991 y 1 es Australiano: Amaurobioides O. Pickard-Cambridge, 1883; el resto, el $86 \%$ de los géneros de la familia, poseen distribución exclusivamente Neotropical.

Pero aunque en la actualidad se trate de una familia con distribución preponderante Neotropical se cree que su origen es Holártico (Legendre, 1979). La familia se originaría en el primitivo continente de Laurasia, extendiéndose posteriormene de América del Norte a América del Sur cuando en el Plioceno se formó el istmo de Panamá, por lo tanto muy posterior a la separación entre América del Sur y África, lo cual explica la no existencia de representantes Etiópicos de la familia. Pensamos por ello que
Anyphaena ha de ser uno de los géneros más antiguos de la familia y su presencia en el Norte de Africa data del Mioceno cuando se formó un puente entre Europa y África y el mar Mediterráneo quedó aislado y casi se deseca, y ya existía el desierto del Sahara impidiendo su dispersión por ese continente.

Por tanto, en Europa, y en concreto en la Península Ibérica y Baleares se encuentra un único género: Anyphaena, del que se reconocen en la actualidad 96 especies con distribución mayoritaria Neártica y Neotropical. Sólo 14 especies de Anyphaena se encuentran en la Región Paleártica, cuya distrubución es la siguiente: $A$. accentuata es la que posee un área de distibución más extensa estando presente por toda Europa y Asia occidental, A. sabina en la Europa mediterránea, A. albirrorata en la Europa mediterránea occidental y $A$. numida en el mediterráneo occidental siendo la única especie del género que ha sido capturada en el norte de África. El resto de las especies poseen distribuciones conocidas muy restringidas: 7 Asiáticas ( 1 especie en Siria, 3 en Japón, 2 en China y 1 en el Himalaya), 3 en centro Europa (2 en Alemania, 1 en Rumania y 1 en la antigua Checoslovaquia).

\section{Resultados}

Familia ANYPHAENIDAE Bertkau, 1878

SINÓNIMOS: Drassidae: Cuni i Martorell, 1880, 1883 y 1889. Drassidae, Subfamilia Clubioninae: Pérez Acosta, 1919.

Clubionidae: Bacelar, 1928, 1933 y 1940; Machado, 1937 y 1949.

Clubionidae, Subfamilia Clubioninae, Grupo Anyphaeneae: Simon, 1897; Fernández Galiano, 1910.

GÉNEROS CITADOS: Anyphaena Sundevall, 1833

\section{ESPECIES CITADAS:}

1. Anyphaena accentuata (Walckenaer, 1802)

2. Anyphaena albirrorata Simon, 1878

3. Anyphaena numida Simon, 1897

4. Anyphaena sabina L. Koch, 1866

Para los caracteres de diagnosis de estas cuatro especies peninsulares consultar Urones et al., 1995.

NOMEN DUBIUM: Anyphaena concolor Bertkau in Viera, 1893: Vieira, 1893; Bacelar, 1928. Nomen dubium: Urones et al., 1995. 


\section{LISTA SISTEMÁTICA DE ESPECIES:}

Anyphaena accentuata (Walckenaer, 1802)

Aranea accentuata: Walckenaer, 1802, Faune parisienne. Insectes ou Histoire abrégée des Insectes des environs de Paris. Paris. 2 vol., p.226

REFERENCIAS BIBLIOGRÁFICAS.- $1,2,4,5,6,8,9$, $12,13,14,15,19,25,26,27,28$

LOCALIDADES CITADAS.- España: BARCELONA: San Miguel del Fay (Cuni i Martorell, 1880; Fernández Galiano, 1910; Pérez Acosta, 1919), La Garriga (Cuni i Martorell, 1883; Fernández Galiano, 1910; Pérez Acosta, 1919), Calella (Cuni i Martorell, 1898; Fernández Galiano, 1910; Pérez Acosta, 1919), Montseny (El Vilar de la Castanya) (Barrientos \& Ascaso, 1985; Barrientos, 1986; Espuny et al., 1993; Urones et al., 1995). CÁCERES: Hervás, Jerte y Valdastillas (Urones \& Puerto, 1988; Urones et al., 1995). GERONA: Arbucias (Cuni i Martorell, 1880; Fernández Galiano, 1910; Pérez Acosta, 1919; Bosmans \& Keer, 1985). HUESCA: Jaca (Alto Aragón occidental) (Duffey, 1983). Jaca (San Juan de la Peña) (Urones, 1985). SALAMANCA: Béjar, Linares de Riofrío (La Honfría), Montemayor del Río, Navasfrías, Peñaparda, Puerto de Vallejera, Villasrubias (Urones \& Puerto, 1988; Urones et al., 1995). ZAMORA: Pías (Urones, 1987).

Portugal: BEIRA ALTA: Guarda (Bacelar, 1928). BEIRA LITORAL: Coimbra (Bacelar, 1928). ESTREMADURA: Marinha Grande, Sintra (Bacelar, 1933).

LOCALIDADES NUEVAS.- España: ÁVILA: Candeleda, 15.04.31, 1 ㅇ (Material del MNCN 3.24/8, 87 -255). CUENCA: Tragacete, 15.06.82, 1 ( (que hizo la puesta en el tubo de captura) y 5 juveniles, batiendo vegetación de Buxus sempervirens que bordeaba un curso de agua seco (C. Urones leg.). HUESCA: Ainsa (La Peña Montañesa), 13.08.83, 2 juveniles, batiendo vegetación de Buxus sempervirens, Juniperus y Fagus sylvatica (C. Urones leg.). MADRID: Aldea del Fresno, 10.03.73, $4 O^{\circ} O^{\circ}$ subadultos y 4 \& 9 subadultas.(S. Pérez leg.). SALAMANCA: Puerto de las Mezas (Navasfrías), 30.08.82, 1 juvenil (C. Urones leg.). SEGOVIA: La Granja de San Ildefonso, 1 (Material del MNCN 3.24/8, 86 250, Mazarredo leg.). ZAMORA: Lubián (Hedroso), 19.07.86, 1 juvenil (C. Urones leg.)

DisTRIBUCIÓN.- Mapa 1.

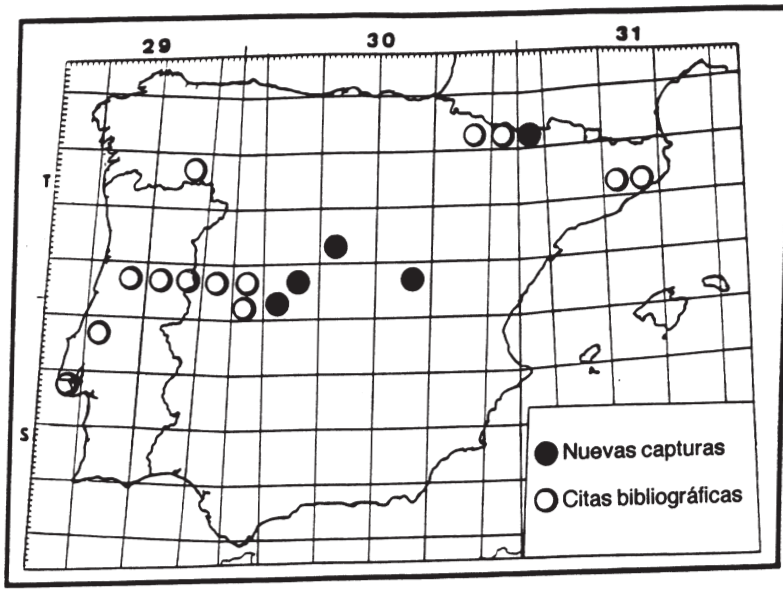

Mapa 1.- Anyphaena accentuata (Walckenaer, 1802).

Map 1.- Anyphaena accentuata (Walckenaer, 1802).

Comentarios.- Los ejemplares de Segovia: La Granja, estaban mal determinados como Anyphaena sabina, y como tal fue citada por Fernández Galiano, 1910. En el artículo de Urones et al. (1985) aparece por error la localidad de La Garriga como perteneciente a la provincia de Gerona, cuando pertenece a Barcelona y no aparece la cita de Arbucias en Gerona.

Se ha recogido por primera vez de las provincias del centro peninsular: Ávila, Cuenca, Madrid y Segovia, lo cual amplia notablemente la distribución de esta especie en el interior de la Península.

Distribución GeOgRÁFICA.- Su área de distribución abarca Europa, ampliamente citada en casi todos los países, y Asia occidental (Simon, 1932; Mikhailov \& Fet, 1986; Dunin, 1989). En la Península ha sido recogida en la mitad norte, siendo la cita más meridional la portuguesa de Sintra.

HÁBITAT.- Vive siempre sobre vegetación arbórea y arbustiva variada: bosques de galerías de río, robledales, encinares montanos, etc. Distribución altitudinal amplia desde los 500 a los 1600 metros.

Fenología.- Los adultos aparecen desde el invierno a comienzos del verano (de diciembre a junio). La cópula la realizan principalmente durante la primavera y las hembras hacen su puesta en el verano entre la vegetación, por lo que aunque coinciden en esa época con los adultos de A. sabina, al ocupar hábitats distintos no muestran competencia entre ellas. Especie de ciclo anual (Urones et al., 1985). 


\section{Anyphaena albirrorata Simon, 1878}

Anyphaena alboirrorata: Simon, 1878, Les Arachnides de France, Tome IV. Roret. Paris, p. 169

SiNÓNIMOS.- Anyphaena albo-irrorata: Machado, 1949. Anyphaena alboirrorata: Bristowe, 1952; Orghidan et al., 1975. Según Bonnet (1955, pág. 342) en esta especie debe aplicarse la regla de supresión del hiato y transformar alboirrorata, como su autor defendía (Simon, 1932) por albirrorata. 28.

REFERENCIAS BIBLIOGRÁFICAS.- 7, 17, 18, 25, 27,

LocAlidades CITADAS.- España: CÁCERES: Descargamaría, Gata y Puerto de Perales (Urones \& Puerto, 1988; Urones et al., 1995). HUESCA: Jaca (San Juan de la Peña) (Urones, 1985). SALAMANCA: El Maíllo (Urones \& Puerto, 1988; Urones et al., 1995)

Baleares: MALLORCA: Sin especificar (Bristowe, 1952); Galilea y Palma de Mallorca (Orghidan et al., 1975)

Portugal: BEIRA ALTA: Guarda (Machado, 1949).

LOCALIDADES NUEVAS.- España: CÁCERES: Navalmoral de la Mata, 10.04.93, 2 우 우 dentro de bolsas de seda construidas entre piedras de un pinar de Pinus pinaster, los ejemplares estaban muertos y sin crías (C. Urones leg.). JAÉN: Mancha Real, 8.04.80, 1 우, en una cuneta de encinar (M.A. Ferrández leg.). MADRID: Madrid (Casa de Campo), 18.03.73, 8 우 우 (M. del Pino leg.). VALLADOLID: Peñafiel, 8.06.85, 1 ㅇ muerta dentro de una bolsa en el suelo de un pinar de Pinus pinaster (C. Urones leg.)

DisTRIBUCIÓN.- Mapa 2

COMENTARIOS.- Se ha recogido por primera vez de las provincias de Jaén, Madrid y Valladolid, constituyendo la localidad de Mancha Real (Jaén) la cita más meridional para la especie en la Península.

DistRIBUCión GEOGRÁfiCA.- Su área de dispersión está reducida a la región Mediterránea occidental europea: Ha sido colectada en España, en los Pirineos orientales franceses (Simon, 1878 y 1932; Denis, 1933; Bosmans \& Keer, 1985) y en la región de Carnia en Italia (Bonnet, 1955). La localidad de Mancha Real (Jaén) es la más meridional conocida para la especie. Esta especie es el único Anyphaenidae que ha sido recogido en las islas Baleares.

HÁBITAT.- Vive sobre vegetación de zonas secas y abiertas, como brezales, pinares abiertos, encinares, etc. y en la época de la puesta se encuentran las

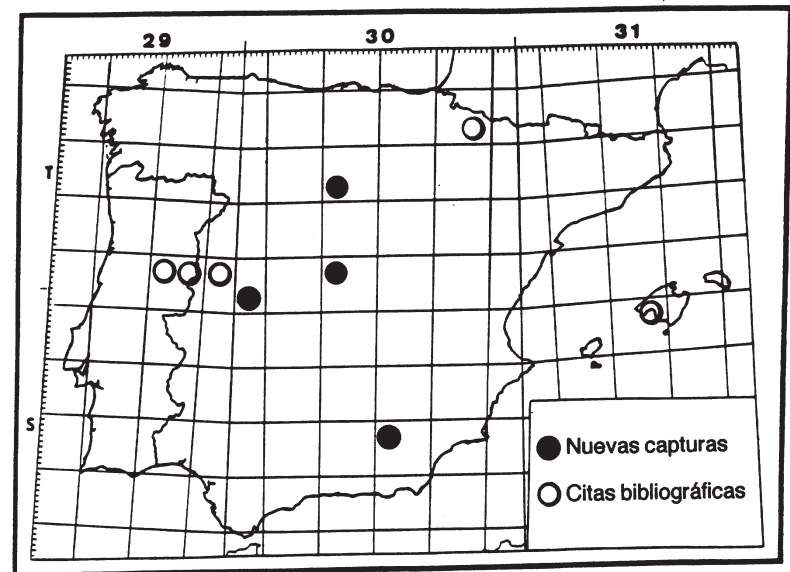

Mapa 2.- Anyphaena albirrorata Simon, 1878.

Map 2.- Anyphaena albirrorata Simon, 1878.

hembras bajo piedras. Distribución altitudinal desde los 280 a los 1000 metros.

FENOLOGíA.- Los adultos aparecen a finales de verano al invierno (preferentemente de octubre a enero), teniendo lugar las cópulas en otoño- invierno. Las hembras se pueden capturar durante la primavera bajo piedras y lugares protegidos, en el interior de bolsas de seda donde se encierran con sus huevos. Se separan ecológicamente de A. numida por preferir ambientes más secos.

\section{Anyphaena numida Simon, 1897}

Anyphaena numida: Simon, 1897, An. Soc. ent. Fr., 65.p. 508

SinÓNIMOS.- Anyphaena occidentalis, Simon, 1897: Simon,1897 y 1898; Fernández Galiano, 1910; Reimoser, 1919; Bacelar, 1928; Machado, 1937. Nueva sinonimia: Urones et al., 1995.

REFERENCIAS BIBLIOGRÁFICAS.- 1, 14, 15, 16, 20, $22,23,26,27,28$

LOCALIDADES CITADAS.- España: ÁVILA: El Arenal, Candeleda y Monasterio de Chilla (Urones \& Puerto, 1988; Urones et al., 1995). BARCELONA: Montseny (El Vilar de la Castanya) (Espuny et al., 1993; Urones et al., 1995). CÁCERES: Baños de Montemayor, La Garganta, Gata, El Guijo de Santa Barbara, Hervás, Jarilla, San Martín de Trvejo, Valdastillas y Valverde del Fresno (Urones \& Puerto, 1988; Urones et al., 1995). LA CORUÑA: Simon, 1897; Fernández Galiano, 1910; Reimoser, 1919. 
SALAMANCA: Aldeacipreste, Las Batuecas (Monasterio y Río Batuecas), Béjar, Candelario, El Cerro, Martinamor, Montemayor del Río, Puebla de San Medel, Puente del Congosto, Sotoserrano (Urones \& Puerto, 1988; Urones et al., 1995). ZAMORA: Toro (Granja Florencia) (Urones, 1987). Portugal: BEIRA ALTA: La Guarda (Simon, 1897; Bacelar, 1928). DOURO LITORAL: Porto (Simon, 1897; Bacelar, 1928). Cete y Valongo (Simon, 1898; Bacelar, 1928). MINHO: Joane (Braga) (Machado, 1937).

LOCALIDADES NUEVAS.- CÁCERES: Moraleja (Río Arrago), 2.01.96, 1 ㅇ, dentro de una bolsa de seda entre pizarras de un muro de separación de fincas (C.Urones leg.). MADRID: Aldea del Fresno, 10.03.73, 1 ㅇ (S. Pérez leg.). Aranjuez, 11.02.32, 4 hembras (Material del MNCN 3.24/8, 85 -216, D. Peláez leg.). El Escorial, 27.02.71, 1 \& grávida (J.M. Herranz leg.). Madrid, 3 ㅇ $\uparrow$ (Material del MNCN 3.24/8, 85 -210). SALAMANCA: Puerto de Vallejera, $1200 \mathrm{~m}, 28.01 .84,1$ ㅇ (L. Gallardo. leg.). SANTANDER: Puente Viesgo, $20^{\circ} \sigma^{7}$ y 2 juveniles (Material del MNCN 3.24/8, 86 -251a, Bolivar leg.). SEGOVIA: Sepulveda, 14.04.85, 1 \% muerta con numerosas crias dentro de una bolsa construida entre piedras (C.Urones leg.).

DISTRIBUCIÓN.- MAPA 3.

COMENTARIOS.- Se ha recogido por primera vez de las provincias de Madrid, Santander y Segovia.

DisTRIBUCIÓN GEOGRÁFICA.- Mediterráneo occidental. Ha sido citada además de España y Portugal, de los Pirineos orientales franceses (Simon, 1932; Denis, 1933; Bosmans \& De Keer, 1985) y de Argelia (Simon, 1897). Es la única especie de Anyphaenidae encontrada en África.

En la Península, hasta ahora, sólo se ha recogido en la mitad norte.

HÁBITAT.- Vive sobre vegetación arbórea y arbustiva variada: galerías de río, robledales, encinares montanos, etc. La hembra se protege en el suelo bajo piedras, cúpulas de bellotas, etc. dentro de una bolsa de seda con su puesta.

Esta especie que posee un gradiente altitudinal amplio: desde los 200 a los 1300 metros.

FenOlogía.- Los adultos aparecen a durante el verano y comienzos del otoño (de julio a septiembre). La cópula sucede principalmente en septiembre, y la hembra se protege entre piedras o cortezas en el interior de una bolsa que teje y allí hace la puesta, no compitiendo con los ejemplares de $A$. albirrorata por ocupar hábitats con mayor humedad ambiental. Los pequeños juveniles se dispersan entre la vegetación en primavera pero para las hem-

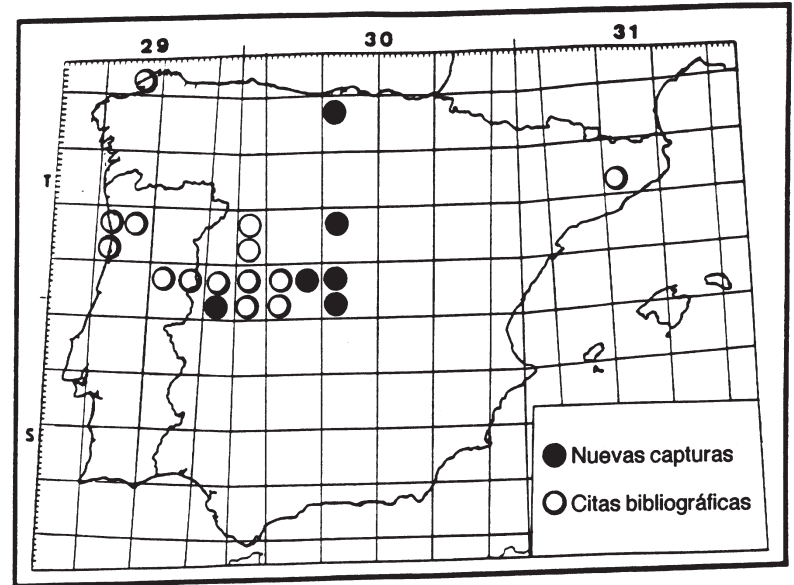

Mapa 3.-_ Anyphaena numida Simon, 1897.

Map 3.- Anyphaena numida Simon, 1897.

bras su bolsa les sirve de ataúd, mueren dentro por lo que no vuelven a salir de su bolsa. Aunque hemos encontrado muchas veces bolsas con hembras muertas y mudas de las pequeñas arañas, en ningún caso hemos visto signos de canibalismo entre las jóvenes arañas y sus progenitoras. Especie de ciclo anual (Urones et al., 1985).

Anyphaena sabina L. Koch, 1866

Anyphaena sabina: L. Koch, 1866, Die Arachniden Familie der Drassiden. Heft 1-6. Nürnberg, pp. 195, 214

REFERENCIAS BIBLIOGRÁFICAS.- $1,3,6,10,11$, $19,23,24,27,28$

LOCALIDADES CITADAS.- España: Sin especificar (Simon, 1878; Fernández Galiano, 1910). ÁVILA: Piedrahita (Urones \& Puerto, 1988; Urones et al., 1995). BARCELONA: Montserrat (Cuni i Martorell, 1889; Fernández Galiano, 1910; Pérez Acosta, 1919), Montseny (El Vilar de la Castanya) (Espuny et al., 1993; Urones et al., 1995), Serra de l' Obac (Urones et al., 1995). CÁCERES: Hervás, San Martín de Trevejo (Urones \& Puerto, 1988; Urones et al., 1995). GERONA: Martorell de la Selva (Empalme) (Cuni i Martorell, 1885; Fernández Galiano, 1910; Pérez Acosta, 1919; Bosmans \& Keer, 1985). SALAMANCA: Las Batuecas (Río Batuecas), Fuenteguinaldo (Urones \& Puerto, 1988; Urones et al., 1995). Martinamor (Urones et al., 1995). 


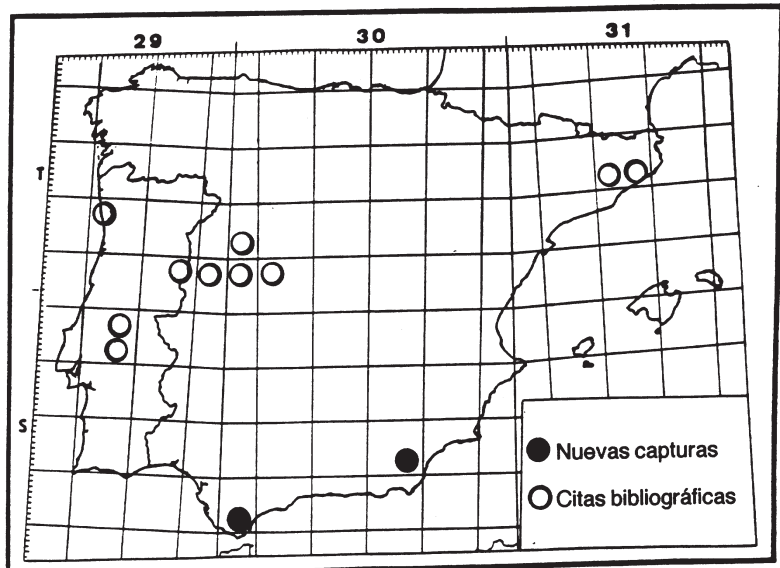

Mapa 4.- Anyphaena sabina L. Koch, 1866.

Map 4.-Anyphaena sabina L. Koch, 1866.

Portugal: Sin especificar (Simon, 1932). ALTO ALENTEJO: Aviz (Portalegre) y Coutada da AreiaCrato (Portalegre) (Bacelar, 1940). DOURO LITORAL: Foz do Douro (Porto) (Simon, 1898; Bacelar, 1928).

LOCALIDADES NUEVAS.- CÁDIZ: Los Barrios (La

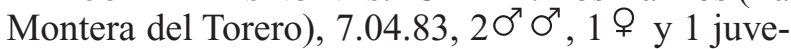
nil, batiendo vegetación de Quercus suber, Ulex y Erica (C.Urones leg.). GRANADA: Jeres del Marquesado (Subida al Puerto de la Ragua, 1600 m), 23.07.96, 1 우 y 1 juvenil, batiendo vegetación de Alnus glutinosa bordeando un arroyo (C.Urones leg.). SALAMANCA: Cabrerizos (La Flecha) 9.06.84, 1 \% , sobre encinas en una vaguada (C.Urones leg.).

DisTRIBUCIÓN.- Mapa 4

COMENTARIOS.- Los ejemplares recogidos en Granada: Jeres del Marquesado, poseeen una coloración muy oscura que podría corresponder a una forma melánica también descrita en Anyphaena accentuata (Roberts, 1985).

Fue citada de Segovia: La Granja (Fernández Galiano, 1910) pero en la revisión del material hemos visto que estaba mal determinado tratándose en realidad de Anyphaena accentuata.

Nuestras capturas aportan las localidades más meridionales para la distribución de la especie.

DisTRIBUCIÓN GEOGRÁFICA.- Su área de distribución comprende la Europa mediterránea. Ha sido recogida, además de en España y Portugal, en Francia, Córcega, Italia, Corfu (Dalmas, 1922; Simon, 1932; Bristowe, 1935; Brignoli \& Gaddini,
1979) y en el Caucaso (Dunin, 1989). La cita más meridional es la española de Los Barrios (Cádiz).

HÁBITAT.- Vive sobre vegetación arbórea y arbustiva variada: tanto robledales como encinares montanos, etc. Distribución altitudinal amplia desde los 150 a los 1600 metros.

FenOlogía.- Los adultos aparecen a finales de invierno, presentándose principalmente en primavera y comienzos de verano, cuando suceden la cópula y la puesta. Coincidiendo su época de desarrollo con los adultos de $A$. accentuata, pero al ocupar hábitats distintos no muestran competencia entre ellos.

\section{AGRADECIMIENTOS}

Quiero hacer constar mi gratitud al Museo Nacional de Ciencias Naturales de Madrid por el préstamo para revisión de los Clubionidae depositados en su colección y que nos han ayudado en la elaboración de este trabajo.

\section{Referencias Bibliográficas Ibéricas}

Para la realización de este catálogo se han consultado numerosas fuentes bibliográficas, las que han aportado datos sobre los Anyphaenidae ibéricos se exponen en la lista siguiente. El número que las precede es el que se utiliza como referencia en el texto.

1. Bacelar, A., 1928. Aracnídios portuguêses III. Catálogo Sistemático dos Aracnídios de Portugal citados por diversos autores (1831-1926). Bull. Soc. portug. sci. nat., 10(17): 169-203.+ Arq. Mus. Bocage, 1(1930): 169-203.

2. BaCElar, A., 1933. Aracnídios portuguêses IV. Bull. Soc. portug. sci. nat., 11(28): 295-306.+ Arq. Mus. Bocage, 4(1933): 295-306.

3. Bacelar, A., 1940. Aracnídios portuguêses VI. (Continuaçào do Inventario dos Aracnídios). Bull. Soc. portug. sci. nat., 13 (20): 99-110 + Arq. Mus. Bocage, 11 (1940): 121-134.

4. Barrientos, J.A., 1986. Aranyes del Montseny. In Terradas, J. \& J. Miralles (Eds.): El patrimoni biològic del Montseny. Catàlegs de flora i fauna, Diputació de Barcelona. Servei de Parcs Naturals. 1: 95-99.

5. Barrientos, J.A. \& Ascaso, C., 1985. Algunas aranas del Montseny. In J. Real (Ed.): El Medi Natural del Vallés.Actas del I Col.loqui de Naturalistes Vallesans. Sabadell: 98-108.

6. Bosmans, R. \& DE Keer, R., 1985. Catalogue des Araignées des Pyrénées. Espèces citées, nouvelles récoltes, et bibliographie. Doc. trav. I.R.S.N.B., $\mathrm{n}^{\circ}$ 23. Bruxelles. 
7. Bristowe, W.S., 1952. The spiders of Islands. $S$. East. Nat., 57: 34-43.

8. Cuni i Martorell, M., 1880. Excursión entomológica y botánica a San Miguel del Fay, Arbucias y cumbres del Montseny. An. Soc. esp. Hist. Nat., 9: 205242.

9. Cuni i Martorell, M., 1883. Resultado de una exploración entomológica y botánica por el término de la Garriga (Cataluña). An. Soc. esp. Hist. Nat., 12 (1): 83-101.

10. Cuni i Martorell, M., 1885. Excursión entomológica a varias localidades de la provincia de Gerona (Cataluña). An. Soc. esp. Hist. Nat., 14(1): 51-73.

11. Cuni i Martorell, M., 1889. Misceláneas entomológicas. Arácnidos de Amer y Montserrat. An. Soc. esp. Hist. Nat., 18(3): 295-299.

12. Cuni i Martorell, M., 1898. Fauna entomológica de la villa de Calella (Cataluña). An. Soc. esp. Hist. Nat., 26: 281-339.

13. Duffey, E., 1983.- Nota preliminar sobre arañas del alto Aragón occidental. Pirineos Jaca, 118: 41-48.

14. Espuny, A., Barrientos, J.A. \& Ascaso, C., 1993. Arañas de un encinar montano (Montseny, Barcelona, España). Resultados faunínsticos. Boll. Acc. Gioenia Sci. Nat. Catania, 26(345): 93-105.

15. Fernández Galiano, E., 1910. Datos para el conocimiento de la distribución geográfica de los Arácnidos en España. Mem. Soc. esp. hist. nat., 6(5): 343-424.

16. Machado, A. Do B., 1937. Aranhas novas para a faun portuguesa (I). Mem. estud. Mus. zool. Univ. Coimbra, ser. I, 107: 1-7.

17. Machado, A. Do B., 1949. Araignées nouvelles pour la faune portugaise (III). Mem. estud. Mus. zool. Univ. Coimbra, 191: 1-69.

18. Orghidan, T., Dumitresco, M. \& Georgescu, M., 1975. Mission biospéologique "Constantin Dragan" à Majorque (1970-1971). Première note Arachnides (Araneae et Pseudoscorpionidea). Trav. Inst. Spéleol. E. Racovitza, Bucarest, 14: 9-33.

19. Pérez Acosta, F., 1919. Los arácnidos de Cataluña. (Catálogo sistemático crítico). Treb. Inst. Catal. hist. nat., 6: 9-72.

20. Reimoser, E., 1919. Katalog der echten Spinnen (Araneae) des Paläarktischen Gebietes. Abh. zool.bot. Ges. Wien, 10(2): 1-280.

21. Simon, E., 1878. Les Arachnides de France, Tome IV. Roret. Paris.

22. Simon, E., 1897. Etudes Archnologiques 27, Memoire XLII. Descrptions d' espèces nouvelles de l'ordre des Araneae. An. Soc. ent. Fr., 65: 465-510.

23. Simon, E., 1898. Sur quelques Arachnides du Portugal appartenant au Musée de zoologie de l'Académie polytechnique de Porto. Ann. sci. nat. Porto , 5: 92-102.
24. Simon, E., 1932. Les Arachnides de France, Tome.VI, 4éme partie. Roret. Paris. 773-978 pp.

25. Urones, C., 1985. Artrópodos epigeos del Macizo de San Juan de la Peña (Jaca, prov. de Huesca). VII. arañas clubionoideas. Pirineos, Jaca, 126: 43-59.

26. Urones, C., 1987. Distribución y ecología de las Arañas en la provincia de Zamora. Anuario de 1986 del Instituto de Estudios Zamoranos Florián de Ocampo: Excma. Diputación de Zamora: 67-122.

27. Urones, C. \& Puerto, A., 1988. Ecological study of the Clubionoidea and Thomisoidea (Araneae) in the Spanish Central System. Rev. Arachnol., 8(1): 1-32.

28. Urones, C., Barrientos, J.A. \& Espuny, A., 1995. El género Anyphaena Sundevall, 1833 (Araneae: Anyphaenidae) en la Península Ibérica. Boln. Asoc. esp. Ent., 19(1-2): 109-131.

29. VieIRA, 1893. Nova lista de especies de aranhas de Portugal, classificadas pelo Dr. Ph. Bertkau, de Bohn, e colligidas pelo Sr. A.F.Moller también esistentes no Museo da Universidade de Coimbra. Instituto, 40(12): 924-926.

\section{Otra bibliografía citada}

Bonnet, P., 1955. Bibliographia Araneorum (Analyse méthodique de toute la littérature araneologique jusqu'en 1939). Tome II ( $1^{\mathrm{a}}$ partie). Toulouse, Douladoure, $918 \mathrm{pp}$.

Brescovit, 1993. Aranhas do gênero Hibana Brescovit: Espécie nova, combinaçòes, sinonímias e novas ocorrências para a regiào Neotropical (Araneae, Anyphaenidae). Rev. bras. Ent., 37(1): 131-139.

BRignoli, P.M., 1983. A Catalogue of the Araneae described between 1940 and 1981. Ed. Merret. Manchester Univ. Press \& British Arach. Soc.: 755 pp.

Brignoli, P.M. \& GAdDini, A., 1979. Nuovi dati su alcuni Anyphaenidae, Liocranidae e Gnaphosidae italiani (Araneae). Boll. Ass. Romana entomol., 34: 10- 15.

BRISTOWE, W.S. 1935. The Spiders of Greece and the adjacents Islands. Proc. Zool. Soc. London: 733- 788.

Coddington, J.A. \& LeVi, H.W., 1991. Systematics and evolution of spiders (Araneae). Annu. Rev. Ecol. Syst., 22: 565- 592.

DAlmas, R. DE C., 1922. Catalogue des araignées récoltées par le Marquis G. Doria dans l' île Giglio (Archipel toscan). Ann. Mus. civ. stor. nat. Genova, 50: 79-96.

Denis, J., 1933. Chasses Arachnologiques dans les Pyrénées Orientales (Région de Banyuls-sur-mer et Vallespir). Bull. Soc. Hist. nat. Toulouse, 65: 529-591.

Dondale, C.D. \& Redner, J.H., 1982. The insects and arachnids of Canada. Part 9. The sac spiders of Canada and Alaska (Araneae: Clubionidae and Anyphaenidae). Can Dep. Agr. Publicationes. Ottawa. $194 \mathrm{pp}$ 
DuNIN, P.M., 1989.- Fauna and altitudinal distribution of spiders (Arachnida, Aranei) of teh Azerbaijan part of the southern macroslope of the Caucasus Major . In A.B. Lange (Ed.) Fauna and Ecology of spiders and scorpions. Arachnological collected papers. Nauka Publ. Moscow: 31-39.

LEGENDRE, R., 1979. Les araignées et la dérive des continents austraux. Ann. Biol., 18(1-2): 37- 68.

LeHTinen, P.T., 1967. Classification of the Cribellate spiders and some allied families, with notes on the evolution of the suborder Araneomorpha. Ann. Zool. Fenn., 4: 199-468.

Mikhailov, K.G. \& JA Fet, V., 1986. Materials to the spider fauna (Aranei) of Turkmenia, I. Families Anyphaenidae, Sparassidae, Zoridae, Clubionidae, Micaridae, Oxyiopidae. In: Fauna, systematika i filogeniya bespozvonochnykh zhivotnykh. Sbornik trudov Zool. Muzeya Mosk. Gos. Univ.: 24: 168-186.

Platnick, N.I., 1989. Advances in Spider Taxonomy 1981-1987: A Supplement to Brignoli's A Catalogue of the Araneae described between 1940 and 1981. Manchester University Press. 673 pp.

Platnick, N.I., 1993. Advances in Spider Taxonomy 1988-1991: With Synonymies and Transfers 1940 1980. The New York Entomological Society. 846 pp.
RoBerTs, M.J., 1985. The Spiders of Great Britain and Ireland. vol. I. Harley Books, Colchester, England. $229 \mathrm{pp}$.

Urones, C. 1995 (1996). Catálogo y atlas de las arañas de la familia Philodromidae Thorell, 1870 de la Península Ibérica e Islas Baleares. Graellsia, 51: 5581.

Recibido, el 26-XII-1996 Aceptado, el 24-IX-1997 Publicado, el 31-X-1997

\section{Apéndice}

Relación de localidades que han suministrado nuevas citas de Anyphaenidae, agrupadas por provincias, con sus características principales.

\begin{tabular}{|c|c|c|c|c|}
\hline Provincia & $\underline{\text { LOCALIDAD }}$ & U.T.M. & ALTITUD (m) & VEGETACIÓN \\
\hline Ávila & Candeleda & 30TUK0848 & 500 & Quercus pyrenaica \\
\hline \multirow[t]{2}{*}{ Cáceres } & Moraleja (Río Arrago) & 29TPE9935 & 300 & Quercus rotundifolia \\
\hline & Navalmoral de la Mata & 30STK72 & 280 & Pinus pinaster \\
\hline Cádiz & Los Barrios (La Montera del Torero) & 30STF71 & 150 & Quercus suber, Ulex, Erica \\
\hline Cuenca & Tragacete & 30TWK96 & \pm 1.200 & Buxus sempervirens \\
\hline \multirow[t]{2}{*}{ Granada } & Jeres del Marquesado (Subida al & & & \\
\hline & Puerto de la Ragua) & 30TVG8310 & 1.600 & Alnus glutinosa \\
\hline Huesca & Ainsa (La Peña Montañesa) & 31TBH6908 & 1.600 & $\begin{array}{l}\text { Buxus sempervirens, Fagus sylvatica, } \\
\text { Juniperus. }\end{array}$ \\
\hline Jaén & Mancha Real & 30TVG48 & 750 & Quercus rotundifolia \\
\hline \multirow[t]{4}{*}{ Madrid } & Aldea del Fresno & 30TUK96 & 500 & - \\
\hline & Aranjuez & 30TVK43 & 500 & - \\
\hline & El Escorial & 30TVK09 & \pm 900 & - \\
\hline & Madrid (La Casa de Campo) & 30TVK47 & 650 & - \\
\hline \multirow[t]{4}{*}{ Salamanca } & Cabrerizos (La Flecha) & 30TTL8140 & 790 & Quercus rotundifolia \\
\hline & Navasfrías (Puerto de las Mezas) & 29TPE8359 & 1.100 & Quercus pyrenaica \\
\hline & Vallejera de Riofrío (Puerto & & & \\
\hline & de Vallejera) & 39TTK7078 & 1.200 & Quercus pyrenaica \\
\hline Santander & Puente Viesgo & $30 \mathrm{TVN} 29$ & \pm 200 & - \\
\hline \multirow[t]{2}{*}{ Segovia } & La Granja de San Ildefonso & 30TVL12 & 1.200 & - \\
\hline & Sepulveda & 30TVL37 & 920 & 一 \\
\hline Valladolid & Peñafiel & 30TVM00 & 750 & Pinus pinaster \\
\hline Zamora & Lubián (Hedroso) & 29TPG7557 & 1.100 & - \\
\hline
\end{tabular}

